\title{
$\mathrm{BMJ}$
}

\section{Sociodemographic patterning of non-communicable disease risk factors in rural India: a cross sectional study}

\author{
Sanjay Kinra, senior lecturer, ${ }^{1}$ Liza J Bowen, research degree student, ${ }^{1}$ Tanica Lyngdoh, research fellow, ${ }^{2}$ \\ Dorairaj Prabhakaran, adjunct professor, ${ }^{2}$ Kolli Srinath Reddy, president, ${ }^{3}$ Lakshmy Ramakrishnan, associate \\ professor, ${ }^{4}$ Ruby Gupta, research fellow, ${ }^{4}$ Ankalmadagu V Bharathi, lecturer, ${ }^{5}$ Mario Vaz, senior lecturer, ${ }^{5}$ \\ Anura V Kurpad, professor, ${ }^{5}$ George Davey Smith, professor, ${ }^{6}$ Yoav Ben-Shlomo, professor, ${ }^{6}$ Shah Ebrahim, \\ professor $^{1}$
}

${ }^{1}$ Non-communicable Disease Epidemiology Unit, London School of Hygiene and Tropical Medicine, London WC1E 7HT, UK

${ }^{2}$ Centre for Chronic Disease Control, New Delhi, India

${ }^{3}$ Public Health Foundation of India, New Delhi, India

${ }^{4}$ Department of Biochemistry, All India Institute of Medical Sciences, Delhi, India

${ }^{5}$ St John's Research Institute, Bangalore, India

${ }^{6}$ Department of Social Medicine, University of Bristol, Bristol

Correspondence to: S Kinra sanjay.kinra@lshtm.ac.uk

Cite this as: BMJ 2010;341:c4974 doi:10.1136/bmj.c4974

\section{ABSTRACT}

Objectives To investigate the sociodemographic patterning of non-communicable disease risk factors in rural India.

Design Cross sectional study.

Setting About 1600 villages from 18 states in India. Most were from four large states due to a convenience sampling strategy.

Participants 1983 (31\% women) people aged 20-69 years (49\% response rate).

Main outcome measures Prevalence of tobacco use, alcohol use, low fruit and vegetable intake, low physical activity, obesity, central adiposity, hypertension, dyslipidaemia, diabetes, and underweight.

Results Prevalence of most risk factors increased with age. Tobacco and alcohol use, low intake of fruit and vegetables, and underweight were more common in lower socioeconomic positions; whereas obesity, dyslipidaemia, and diabetes (men only) and hypertension (women only) were more prevalent in higher socioeconomic positions. For example, 37\% (95\% Cl 30\% to $44 \%$ ) of men smoked tobacco in the lowest socioeconomic group compared with $15 \%$ (12\% to $17 \%)$ in the highest, while $35 \%$ (30\% to $40 \%$ ) of women in the highest socioeconomic group were obese compared with $13 \%(7 \%$ to $19 \%)$ in the lowest. The age standardised prevalence of some risk factors was: tobacco use $(40 \%$ ( $37 \%$ to $42 \%$ ) men, $4 \%$ (3\% to $6 \%$ ) women); low fruit and vegetable intake (69\% (66\% to $71 \%)$ men, $75 \%$ ( $71 \%$ to $78 \%$ ) women); obesity (19\% (17\% to $21 \%$ ) men, $28 \%$ (24\% to $31 \%$ ) women); dyslipidaemia (33\% (31\% to $36 \%$ ) men, $35 \%$ (31\% to 38\%) women); hypertension ( $20 \%$ (18\% to $22 \%$ men, $22 \%$ (19\% to $25 \%$ ) women); diabetes (6\% (5\% to $7 \%$ ) men, $5 \%$ ( $4 \%$ to $7 \%$ ) women); and underweight (21\% (19\% to $23 \%$ ) men, $18 \%$ (15\% to $21 \%$ ) women). Risk factors were generally more prevalent in south Indians compared with north Indians. For example, the prevalence of dyslipidaemia was $21 \%$ (17\% to $33 \%$ ) in north Indian men compared with $33 \%$ (29\% to $38 \%$ ) in south Indian men, while the prevalence of obesity was $13 \%(9 \%$ to $17 \%)$ in north Indian women compared with $24 \%$ (19\% to $30 \%$ ) in south Indian women.
Conclusions The prevalence of most risk factors was generally high across a range of sociodemographic groups in this sample of rural villagers in India; in particular, the prevalence of tobacco use in men and obesity in women was striking. However, given the limitations of the study (convenience sampling design and low response rate), cautious interpretation of the results is warranted. These data highlight the need for careful monitoring and control of non-communicable disease risk factors in rural areas of India.

\section{INTRODUCTION}

The current epidemic of non-communicable diseases in India is attributed to increased longevity and lifestyle changes resulting from urbanisation. ${ }^{12}$ However, recent data suggest that non-communicable diseases are already the commonest cause of death in some parts of rural India..$^{3-5}$ This is plausible as, apart from improvements in life expectancy, the greater interconnectedness increasingly allows rural populations to adopt urban lifestyles without migration to urban areas. ${ }^{5-7}$ A rise in the prevalence of non-communicable disease risk factors in rural areas has important public health implications, since, notwithstanding the rapid urbanisation, two thirds of India's one billion population still lives in rural areas. ${ }^{8}$ Rural populations have limited access to health care and can least afford to pay for the high treatment costs associated with chronic conditions.

Several surveys have examined the prevalence of risk factors for non-communicable disease in urban India, but recent data from rural India are sparse. ${ }^{9-12}$ Those that exist are limited to selected locations (invariably chosen within a convenient distance of an urban centre) or risk factors and do not allow systematic examination of nationally representative sociodemographic patterns. ${ }^{513-19}$ Knowing the sociodemographic patterns of non-communicable disease risk factors across rural India is important not only for predicting the future course of the epidemic and planning relevant policies for prevention and disease control, but may also provide new aetiological insights through 
their juxtaposition to known variations in disease patterns (such as higher disease prevalence reported from south India). ${ }^{2021}$

The Indian Migration Study was established to investigate the effects of rural-urban migration by using a sibling pair design to collect data on migrant urban factory workers and their co-resident spouses and their non-migrant rural dwelling siblings. ${ }^{22} \mathrm{We}$ carried out a secondary analysis of data on the rural participants of this study to examine the prevalence of non-communicable disease risk factors by age, sex, socioeconomic position, and geographical location of the participants. We hypothesised that the prevalence of risk factors would increase with age and socioeconomic position, and be relatively higher among men and south Indians.

\section{METHODS}

\section{Study population}

The Indian Migration Study was nested within a cardiovascular risk factor surveillance system, which monitors risk factors in industry populations across several large cities in India. ${ }^{12}$ Factory sites in four large cities (Lucknow, Nagpur, Hyderabad, and Bangalore) were chosen to sample populations from the north, centre, and south of the country. ${ }^{22}$ Factory workers and their co-resident spouses were surveyed using employer records as a sampling frame and recruited to the study if they had migrated from a rural area. Each participant (factory worker or spouse) was asked to invite one non-migrant full sibling of the same sex and closest to them in age still residing in their rural place of origin. Precedence was given to sex over age, and when multiple same sex siblings were available the one closest in age was invited. In a small number of cases where no rural sibling was available $(<5 \%)$, a cousin or a close friend from the same village was invited. There were no other exclusion criteria at this recruitment stage. This convenience sampling strategy resulted in rural dwelling siblings being drawn from anywhere in the country (18 of the 28 states), although a substantial proportion came from the four large states in which the factories were based, reflecting the migration patterns of the factory workers and their spouses. The fieldwork took place between March 2005 and December 2007.

\section{Measurements}

Interviewers administered a set questionnaire to collect demographic and behavioural risk factor data. Participants were asked about their place of residence (village, town, and small or big city), years of formal education (none, primary ( 1 to $\leq 4$ years), secondary ( 5 to $\leq 12$ years), and beyond secondary), and occupation (housework, unemployed, manual, skilled manual, and non-manual or professional). ${ }^{23}$ Data on socioeconomic position was collected through a subset of questions used to derive the standard of living index, which is a household level, asset based scale (covering quality of housing and ownership of land and durable goods) devised for use in India. ${ }^{24}$ The index is an appropriate measure of socioeconomic position in rural India, where the joint family structure of the household renders an individual's own socioeconomic position less important. The full index has a large number of items (29 in total), but we restricted this to 13 items by selecting a priori the ones we believed to be most informative. We subsequently validated the short index against the full standard of living index using the dataset of the second National Family Health Survey NFHS-2 (the national demographic survey of India involving 91117 households). ${ }^{24}$ The short index classified $98.5 \%$ ( $n=89716)$ of the survey participants in the same or adjacent fifth of the full index $(66 \%$ in the same fifth), with only $1.5 \%(\mathrm{n}=1401)$ falling outside this range ( $\kappa$ statistic 0.58 ); there was no evidence for an urban-rural bias in classification.

Participants were asked about their current tobacco use in any form (smoked or chewed on a daily basis in the previous six months), and regular consumption of alcohol (on $\geq 10$ days a month in the previous six months).

A quantitative food frequency questionnaire was developed, based on methods described elsewhere ${ }^{25}$ and used to collect data on food intake (including fruit and vegetables) over the previous year. For seasonal foods, intake during the appropriate seasons was estimated. The questionnaire was developed to cover the different dietary behaviours in all of the regions included in the study, and specific recipes for regions and rural and urban settings were collected to account for differences in food preparation between areas.

A quantitative physical activity questionnaire specific to the Indian population was developed using methods described previously, ${ }^{22}$ and subsequently validated against reference methods in both rural and urban Indians. ${ }^{26}$ The questionnaire is used to gather information on participants' habitual daily physical activity based on involvement in potential active and passive activities (occupational, household, hobby, exercise, sedentary, travel, etc) and their duration and frequency over the previous month. Each reported activity was assigned a MET (metabolic equivalent of task) score based on the Compendium of Physical Activities, which is the energy cost of the activity expressed as multiples of the basal metabolic rate (energy required for essential physiological functioning). ${ }^{27}$ For example, a MET score of 3.3 for walking (at moderate pace on a level ground) suggests that 3.3 times more energy is used in walking compared with lying down and resting. The MET score of each activity was multiplied by its duration (hours) and summed over a 24 hour period to estimate the total MET hours per day. Total MET values were used to generate the physical activity level, a ratio of estimated energy expenditure and basal metabolic rate. ${ }^{26}$

Participants were asked whether they had heart disease, high blood pressure, or diabetes; if they had ever had a stroke; and if they were receiving any regular medication for these conditions.

Participants were also asked about their travel time to the study site. 
Table 1|Characteristics of the rural participants of the Indian Migration Study. Values numbers (percentages) of participants

\begin{tabular}{|c|c|c|c|}
\hline & Men $(n=1375)$ & Women $(n=608)$ & Total $(n=1983)$ \\
\hline \multicolumn{4}{|l|}{ Age group (years): } \\
\hline $20-29$ & 268 (19) & $76(13)$ & $344(17)$ \\
\hline $30-39$ & $391(28)$ & $156(26)$ & $547(28)$ \\
\hline $40-49$ & $428(31)$ & $196(32)$ & $624(31)$ \\
\hline $50-59$ & $214(16)$ & $151(25)$ & 365 (18) \\
\hline $60-69$ & $74(5)$ & $29(5)$ & $103(5)$ \\
\hline \multicolumn{4}{|l|}{ Education (years): } \\
\hline No formal education & $167(12)$ & $222(37)$ & $389(20)$ \\
\hline Primary (1-4) & $222(16)$ & $167(27)$ & $389(20)$ \\
\hline Secondary (5-12) & $692(50)$ & $162(27)$ & $854(43)$ \\
\hline Beyond secondary (>12) & $294(21)$ & $57(9)$ & $351(18)$ \\
\hline \multicolumn{4}{|l|}{ Occupation: } \\
\hline Housework & $12(1)$ & $414(68)$ & $426(21)$ \\
\hline Unemployed & $98(7)$ & $27(4)$ & $125(6)$ \\
\hline Manual & $712(52)$ & $96(16)$ & $808(41)$ \\
\hline Skilled manual & 249 (18) & $21(3)$ & $270(14)$ \\
\hline Non-manual or professional & $304(22)$ & $50(8)$ & 354 (18) \\
\hline \multicolumn{4}{|l|}{ Standard of living index*: } \\
\hline Low (0-7) & $147(11)$ & $106(17)$ & $253(13)$ \\
\hline Middle (8-13) & $358(26)$ & $143(24)$ & $501(25)$ \\
\hline High (14-36) & $870(63)$ & $359(59)$ & $1229(62)$ \\
\hline Tobacco use in any form†: & $543(39)$ & $30(5)$ & $573(29)$ \\
\hline Smoked & $277(20)$ & $5(1)$ & $282(14)$ \\
\hline Chewed & $332(24)$ & $26(4)$ & $358(18)$ \\
\hline Alcohol use & $316(23)$ & $35(6)$ & 351 (18) \\
\hline \multicolumn{4}{|l|}{ Geographical region: } \\
\hline North & $752(55)$ & 209 (34) & $961(48)$ \\
\hline South§ & $623(45)$ & $399(66)$ & $1022(52)$ \\
\hline \multicolumn{4}{|l|}{ Travel time to study centre (hours): } \\
\hline Short («4) & $368(27)$ & $171(28)$ & $539(27)$ \\
\hline Medium (4-11) & $659(48)$ & $280(46)$ & $939(47)$ \\
\hline Long ( $\geq 12)$ & $348(25)$ & $157(26)$ & $505(25)$ \\
\hline
\end{tabular}

*Based on subset of questions from the standard of living index. ${ }^{24}$

†Used on a daily basis any time in past 6 months.

$\ddagger$ Consumed on $\geq 10$ days/month any time in the past 6 months.

§Includes the four southern states of Andhra Pradesh, Kerala, Karnataka, and Tamil Nadu.

The participants were weighed in light indoor clothing with a digital weighing machine with $100 \mathrm{~g}$ accuracy (Model PS16, Beurer, Germany). Height was measured in bare feet in the Frankfort plane with a portable plastic stadiometer with a base plate, accurate to $1 \mathrm{~mm}$ (Leicester height measure, Chasmors, London). Waist circumference was measured twice on bare skin at the natural waist (the narrowest part of the abdomen between the ribs and the iliac crest, as seen from the anterior aspect) with a non-stretch metallic tape with a blank lead-in (Chasmors, London). In the absence of a consensus on optimal protocol for waist measurement, this protocol was chosen because it is least invasive and more culturally appropriate. ${ }^{28}$ Blood pressure was measured on the right upper arm in the sitting position, after a rest of 5 minutes. Two readings were taken with an appropriate sized cuff connected to a digital device (Model M5-I, Omron, Matsusaka, Japan), and the mean of the two readings was used for analyses.
Venous blood samples were taken after fasting $(>8$ hours) and centrifuged within 45 minutes, stored locally at $-20^{\circ} \mathrm{C}$, and transported monthly to the Cardiac Biochemistry Laboratory at the All India Institute of Medical Sciences (New Delhi) for biochemical assays. Serum high density lipoprotein (HDL) cholesterol was estimated directly by the elimination method, total cholesterol was estimated by an enzymatic end point method, triglycerides by the GPO-PAP method (Randox Laboratories, Crumlin, UK), and glucose by the GOD-PAP method (Randox Laboratories).

\section{Statistical analyses}

Rural siblings of migrant factory workers and their coresident spouses were included in the analyses. Participants were excluded if they reported their current place of residence as urban (town or city) or were not within the 20-69 year age range. We examined the following potential risk factors for non-communicable disease: current tobacco (smoked or chewed) or alcohol use, low physical activity level $(\leq 1.69),{ }^{26}$ low fruit and vegetable intake $(<400 \mathrm{~g} /$ day, the WHO recommended level), ${ }^{29}$ overweight (using the Asian and WHO cut-off values for body mass index of $\geq 23$ and $\geq 25$, respectively), ${ }^{30}$ abdominal obesity (using modified waist circumference cut-offs appropriate for Indian populations, $>90 \mathrm{~cm}$ for men and $>85 \mathrm{~cm}$ for women), ${ }^{31}$ high ratio of total cholesterol to HDL cholesterol ( $\geq 4.5)$, high triglyceride concentration $(\geq 1.69 \mathrm{mmol} / \mathrm{l}){ }^{32}$ hypertension (self reported or blood pressure $\geq 140 / 90 \mathrm{~mm} \mathrm{Hg}$ ), ${ }^{33}$ diabetes (self reported or fasting blood glucose concentration $\geq 7 \mathrm{mmol} / \mathrm{l}$ ), ${ }^{34}$ underweight (body mass index $<18.5$ ), ${ }^{30}$ and short stature (height $<25$ th centile of the rural population of the National Family Health Survey NFHS- $3^{35}$ ).

Initially the distribution of each risk factor was examined by age (grouped in 10 year bands) and sex. We calculated prevalences with 95\% confidence intervals for binary variables and means with standard deviations for continuous variables; medians (interquartile range) are presented for triglycerides and fasting blood glucose since these data were positively skewed. We assessed trends in age and sex differences by fitting regression models for each outcome and performing Wald tests on model parameters; logistic regression was used for binary variables and linear regression for continuous variables.

We then calculated age adjusted prevalences of risk factors by socioeconomic position (classified into three groups based on standard of living index $)^{24}$ and sex. The full standard of living index uses weights to give a total score of 67 (low 0-14, middle 15-24, high 25-67); we applied the same weights to the data in our short index but proportionately scaled down the cut-offs used in the full index to reflect our total score of 36 (low $0-7$, middle 8-13, high 14-36). Age specific prevalences for 10 year age bands were calculated for each socioeconomic group and sex, and were applied to the age distribution of the total population to estimate age standardised prevalence (standardised internally to the study 
Table 2 |Distribution of risk factors for non-communicable diseases in rural participants of the Indian Migration Study by age and sex. Values are percentages ( $95 \%$ confidence intervals) unless stated otherwise

\begin{tabular}{|c|c|c|c|c|c|c|c|c|c|c|c|c|c|c|c|}
\hline & \multicolumn{6}{|c|}{ Men's age groups (years) } & \multicolumn{6}{|c|}{ Women's age groups (years) } & \multicolumn{3}{|c|}{ All ages } \\
\hline & $\begin{array}{c}20-29 \\
(n=268)\end{array}$ & $\begin{array}{c}30-39 \\
(n=391)\end{array}$ & $\begin{array}{c}40-49 \\
(n=428)\end{array}$ & $\begin{array}{c}50-59 \\
(n=214)\end{array}$ & $\begin{array}{l}60-69 \\
(n=74)\end{array}$ & $\begin{array}{c}P \\
\text { value* }\end{array}$ & $\begin{array}{l}20-29 \\
(n=76)\end{array}$ & $\begin{array}{c}30-39 \\
(n=156)\end{array}$ & $\begin{array}{c}40-49 \\
(n=196)\end{array}$ & $\begin{array}{c}50-59 \\
(n=151)\end{array}$ & $\begin{array}{l}60-69 \\
(n=29)\end{array}$ & $\begin{array}{c}P \\
\text { value }^{*}\end{array}$ & $\begin{array}{c}\text { Men } \\
(n=1375)\end{array}$ & $\begin{array}{l}\text { Women } \\
(n=608)\end{array}$ & $\begin{array}{c}P \\
\text { value* }\end{array}$ \\
\hline $\begin{array}{l}\text { Smoke } \\
\text { tobaccot }\end{array}$ & $\begin{array}{c}7.1 \text { ( } 4.3 \text { to } \\
10.8)\end{array}$ & $\begin{array}{l}18.4(14.7 \\
\text { to } 22.6)\end{array}$ & $\begin{array}{c}22.4(18.6 \\
\text { to } 26.7)\end{array}$ & $\begin{array}{c}34.1(27.8 \\
\text { to } 40.9)\end{array}$ & $\begin{array}{c}23.0(14.0 \\
\text { to } 34.2)\end{array}$ & $<0.001$ & $0(0$ to 4.7$)$ & $0(0$ to 2.3$)$ & $\begin{array}{c}0.5 \text { (0 to } \\
2.8)\end{array}$ & $\begin{array}{c}2.0(0.4 \text { to } \\
5.7)\end{array}$ & $\begin{array}{c}3.4(0.1 \text { to } \\
17.8)\end{array}$ & 0.024 & $\begin{array}{c}20.1(18.1 \\
\text { to } 22.4)\end{array}$ & $\begin{array}{c}0.8(0.3 \text { to } \\
1.9)\end{array}$ & $<0.001$ \\
\hline Chew tobacco† & $\begin{array}{c}28.0(22.7 \\
\text { to } 33.8)\end{array}$ & $\begin{array}{c}28.4(24.0 \\
\text { to } 33.1)\end{array}$ & $\begin{array}{c}20.6(16.8 \\
\text { to } 24.7)\end{array}$ & $\begin{array}{c}20.1(14.9 \\
\text { to } 26.1)\end{array}$ & $\begin{array}{c}20.3(11.8 \\
\text { to } 31.2)\end{array}$ & 0.004 & $0(0$ to 4.7$)$ & $\begin{array}{c}1.9(0.4 \text { to } \\
5.5)\end{array}$ & $\begin{array}{c}3.1 \text { (1.1 to } \\
6.5)\end{array}$ & $\begin{array}{c}8.6(4.7 \text { to } \\
14.3)\end{array}$ & $\begin{array}{l}13.8(3.9 \\
\text { to } 31.7)\end{array}$ & $<0.001$ & $\begin{array}{c}24.1(21.9 \\
\text { to } 26.5)\end{array}$ & $\begin{array}{c}4.3(2.8 \text { to } \\
6.2)\end{array}$ & $<0.001$ \\
\hline Alcohol useł & $\begin{array}{l}11.2(7.7 \\
\text { to } 15.6)\end{array}$ & $\begin{array}{c}23.5(19.4 \\
\text { to } 28.1)\end{array}$ & $\begin{array}{c}24.8(20.7 \\
\text { to } 29.1)\end{array}$ & $\begin{array}{c}31.3(25.2 \\
\text { to } 38.0)\end{array}$ & $\begin{array}{c}28.4(18.5 \\
\text { to } 40.1)\end{array}$ & $<0.001$ & $\begin{array}{c}5.3(1.5 \text { to } \\
12.9)\end{array}$ & $\begin{array}{c}1.9 \text { (0.4 to } \\
5.5)\end{array}$ & $\begin{array}{c}6.1(3.2 \text { to } \\
10.5)\end{array}$ & $\begin{array}{c}9.3(5.2 \text { to } \\
15.1)\end{array}$ & $\begin{array}{c}6.9(0.8 \text { to } \\
22.8)\end{array}$ & 0.043 & $\begin{array}{c}23.0(20.8 \\
\text { to } 25.3)\end{array}$ & $\begin{array}{c}5.8(4.0 \text { to } \\
7.9)\end{array}$ & $<0.001$ \\
\hline $\begin{array}{l}\text { Low physical } \\
\text { activity§ }\end{array}$ & $\begin{array}{c}60.2(54.0 \\
\text { to } 66.1)\end{array}$ & $\begin{array}{c}74.3(69.6 \\
\text { to } 78.6)\end{array}$ & $\begin{array}{c}75.1(70.7 \\
\text { to } 79.1)\end{array}$ & $\begin{array}{c}75.5(69.1 \\
\text { to } 81.1)\end{array}$ & $\begin{array}{l}71.2(59.4 \\
\text { to } 81.2)\end{array}$ & 0.002 & $\begin{array}{c}72.4(60.9 \\
\text { to } 82.0)\end{array}$ & $\begin{array}{c}76.1(68.6 \\
\text { to } 82.6)\end{array}$ & $\begin{array}{c}77.2(70.6 \\
\text { to } 82.9)\end{array}$ & $\begin{array}{c}74.7(66.9 \\
\text { to } 81.4)\end{array}$ & $\begin{array}{c}57.1(37.2 \\
\text { to } 75.5)\end{array}$ & 0.460 & $\begin{array}{c}71.8(69.3 \\
\text { to } 74.2)\end{array}$ & $\begin{array}{c}74.8(71.1 \\
\text { to } 78.2)\end{array}$ & 0.180 \\
\hline $\begin{array}{l}\text { Low fruit and } \\
\text { vegetable } \\
\text { intakef }\end{array}$ & $\begin{array}{c}63.8(57.7 \\
\text { to } 69.6)\end{array}$ & $\begin{array}{c}65.2(60.3 \\
\text { to } 69.9)\end{array}$ & $\begin{array}{l}70.1(65.5 \\
\text { to } 74.4)\end{array}$ & $\begin{array}{c}73.8(67.4 \\
\text { to } 79.6)\end{array}$ & $\begin{array}{c}75.7(64.3 \\
\text { to } 84.9)\end{array}$ & 0.002 & $\begin{array}{c}65.8(54.0 \\
\text { to } 76.3)\end{array}$ & $\begin{array}{c}71.2(63.4 \\
\text { to } 78.1)\end{array}$ & $\begin{array}{c}76.0(69.4 \\
\text { to } 81.8)\end{array}$ & $\begin{array}{c}81.5(74.3 \\
\text { to } 87.3)\end{array}$ & $\begin{array}{c}93.1(77.2 \\
\text { to } 99.2)\end{array}$ & $<0.001$ & $\begin{array}{c}68.4(65.8 \\
\text { to } 70.8)\end{array}$ & $\begin{array}{c}75.7(72.0 \\
\text { to } 79.0)\end{array}$ & 0.001 \\
\hline $\begin{array}{l}\text { Mean (SD) body } \\
\text { mass index } \\
\left(\mathrm{kg} / \mathrm{m}^{2}\right)\end{array}$ & $20.1(2.7)$ & $21.7(3.6)$ & $22.7(4.1)$ & $21.7(3.9)$ & $21.6(3.6)$ & $<0.001$ & $20.5(3.7)$ & $22.6(3.9)$ & $23.2(4.5)$ & $23.7(4.7)$ & $21.8(4.3)$ & $<0.001$ & $21.7(3.8)$ & $22.8(4.4)$ & $<0.001$ \\
\hline $\begin{array}{l}\text { Mean (SD) waist } \\
\text { circumference } \\
(\mathrm{cm})\end{array}$ & $74.3(8.0)$ & $\begin{array}{c}80.0 \\
(10.4)\end{array}$ & $\begin{array}{c}83.9 \\
(11.6)\end{array}$ & $\begin{array}{c}82.9 \\
(11.2)\end{array}$ & $\begin{array}{c}82.4 \\
(11.7)\end{array}$ & $<0.001$ & $70.5(9.1)$ & $74.3(9.1)$ & $\begin{array}{c}76.3 \\
(10.8)\end{array}$ & $\begin{array}{c}78.3 \\
(11.2)\end{array}$ & $\begin{array}{c}75.9 \\
(11.1)\end{array}$ & $<0.001$ & $\begin{array}{c}80.7 \\
(11.2)\end{array}$ & $\begin{array}{c}75.5 \\
(10.5)\end{array}$ & $<0.001$ \\
\hline $\begin{array}{l}\text { Median (IQR) } \\
\text { triglycerides } \\
(\mathrm{mmol} / \mathrm{l})\end{array}$ & $\begin{array}{c}1.15(0.85 \\
-1.63)\end{array}$ & $\begin{array}{c}1.22(0.90 \\
-1.65)\end{array}$ & $\begin{array}{c}1.23(0.92 \\
-1.82)\end{array}$ & $\begin{array}{c}1.31(0.97 \\
-1.87)\end{array}$ & $\begin{array}{c}1.26(0.94 \\
-1.68)\end{array}$ & $<0.001$ & $\begin{array}{c}1.08(0.84 \\
-1.60)\end{array}$ & $\begin{array}{c}1.23(0.96 \\
-1.69)\end{array}$ & $\begin{array}{c}1.29(0.97 \\
-1.79)\end{array}$ & $\begin{array}{c}1.3(0.95- \\
1.79)\end{array}$ & $\begin{array}{c}1.76(1.03 \\
-2.57)\end{array}$ & 0 & $\begin{array}{c}1.23(0.90 \\
-1.74)\end{array}$ & $\begin{array}{c}1.27(0.95 \\
-1.79)\end{array}$ & 0.35 \\
\hline $\begin{array}{l}\text { Mean (SD) HDL } \\
\text { cholesterol } \\
(\mathrm{mmol} / \mathrm{l})\end{array}$ & $\begin{array}{c}1.12 \\
(0.23)\end{array}$ & $\begin{array}{c}1.17 \\
(0.24)\end{array}$ & $\begin{array}{c}1.15 \\
(0.24)\end{array}$ & $\begin{array}{c}1.14 \\
(0.26)\end{array}$ & $\begin{array}{c}1.17 \\
(0.24)\end{array}$ & 0.280 & $\begin{array}{c}1.20 \\
(0.28)\end{array}$ & $\begin{array}{c}1.19 \\
(0.24)\end{array}$ & $\begin{array}{c}1.18 \\
(0.26)\end{array}$ & $\begin{array}{c}1.22 \\
(0.27)\end{array}$ & $\begin{array}{c}1.18 \\
(0.27)\end{array}$ & 0.620 & $\begin{array}{c}1.15 \\
(0.24)\end{array}$ & $1.2(0.26)$ & $<0.001$ \\
\hline $\begin{array}{l}\text { Mean (SD) LDL } \\
\text { cholesterol } \\
(\mathrm{mmol} / \mathrm{l})\end{array}$ & $\begin{array}{c}2.53 \\
(0.86)\end{array}$ & $\begin{array}{c}2.72 \\
(1.00)\end{array}$ & $\begin{array}{c}2.80 \\
(0.98)\end{array}$ & $\begin{array}{c}2.88 \\
(1.10)\end{array}$ & $\begin{array}{c}2.88 \\
(0.97)\end{array}$ & $<0.001$ & $\begin{array}{c}2.60 \\
(0.95)\end{array}$ & $\begin{array}{c}2.84 \\
(0.91)\end{array}$ & $\begin{array}{c}2.99 \\
(1.10)\end{array}$ & $\begin{array}{c}3.07 \\
(1.07)\end{array}$ & $\begin{array}{c}3.33 \\
(0.78)\end{array}$ & $<0.001$ & $\begin{array}{c}2.74 \\
(0.99)\end{array}$ & $\begin{array}{c}2.94 \\
(1.03)\end{array}$ & $<0.001$ \\
\hline $\begin{array}{l}\text { Mean (SD) total } \\
\text { cholesterol } \\
(\mathrm{mmol} / \mathrm{l})\end{array}$ & $\begin{array}{c}4.23 \\
(0.97)\end{array}$ & $\begin{array}{c}4.52 \\
(1.10)\end{array}$ & $\begin{array}{c}4.63 \\
(1.14)\end{array}$ & $\begin{array}{c}4.70 \\
(1.23)\end{array}$ & $\begin{array}{c}4.68 \\
(1.07)\end{array}$ & $<0.001$ & $\begin{array}{c}4.39 \\
(1.13)\end{array}$ & $\begin{array}{c}4.67 \\
(1.07)\end{array}$ & $\begin{array}{c}4.83 \\
(1.23)\end{array}$ & $\begin{array}{c}4.95 \\
(1.17)\end{array}$ & $\begin{array}{c}5.34 \\
(1.02)\end{array}$ & $<0.001$ & $\begin{array}{c}4.53 \\
(1.12)\end{array}$ & $\begin{array}{c}4.79 \\
(1.17)\end{array}$ & $<0.001$ \\
\hline $\begin{array}{l}\text { Total:HDL } \\
\text { cholesterol ratio }\end{array}$ & $\begin{array}{c}3.91 \\
(1.01)\end{array}$ & $\begin{array}{c}3.99 \\
(1.16)\end{array}$ & $\begin{array}{c}4.17 \\
(1.23)\end{array}$ & $\begin{array}{c}4.26 \\
(1.25)\end{array}$ & $\begin{array}{c}4.11 \\
(1.03)\end{array}$ & 0.001 & $\begin{array}{c}3.78 \\
(1.00)\end{array}$ & $\begin{array}{c}4.06 \\
(1.16)\end{array}$ & $\begin{array}{c}4.22 \\
(1.21)\end{array}$ & $\begin{array}{c}4.23 \\
(1.32)\end{array}$ & $\begin{array}{c}4.69 \\
(1.06)\end{array}$ & $<0.001$ & $\begin{array}{c}4.08 \\
(1.17)\end{array}$ & $\begin{array}{c}4.15 \\
(1.21)\end{array}$ & 0.220 \\
\hline $\begin{array}{l}\text { Mean (SD) } \\
\text { systolic BP } \\
(\mathrm{mm} \mathrm{Hg})\end{array}$ & $\begin{array}{l}116.7 \\
(11.5)\end{array}$ & $\begin{array}{l}119.2 \\
(12.7)\end{array}$ & $\begin{array}{l}123.1 \\
(17.3)\end{array}$ & $\begin{array}{l}128.4 \\
(21.8)\end{array}$ & $\begin{array}{l}139.4 \\
(24.5)\end{array}$ & $<0.001$ & $\begin{array}{l}108.0 \\
(9.9)\end{array}$ & $\begin{array}{l}115.5 \\
(14.8)\end{array}$ & $\begin{array}{l}121.7 \\
(18.4)\end{array}$ & $\begin{array}{l}131.1 \\
(22.0)\end{array}$ & $\begin{array}{l}131.5 \\
(22.6)\end{array}$ & $<0.001$ & $\begin{array}{l}122.4 \\
(17.3)\end{array}$ & $\begin{array}{l}121.2 \\
(19.5)\end{array}$ & 0.160 \\
\hline $\begin{array}{l}\text { Mean (SD) } \\
\text { diastolic BP } \\
(\mathrm{mm} \mathrm{Hg})\end{array}$ & $72.8(8.8)$ & $\begin{array}{c}76.0 \\
(10.3)\end{array}$ & $\begin{array}{c}77.8 \\
(11.7)\end{array}$ & $\begin{array}{c}79.0 \\
(12.6)\end{array}$ & $\begin{array}{c}81.4 \\
(12.0)\end{array}$ & $<0.001$ & $68.8(7.9)$ & $75.6(9.7)$ & $\begin{array}{c}78.8 \\
(11.6)\end{array}$ & $\begin{array}{c}80.5 \\
(12.0)\end{array}$ & $\begin{array}{l}78.7 \\
(12.9)\end{array}$ & $<0.001$ & $\begin{array}{l}76.7 \\
(11.2)\end{array}$ & $\begin{array}{l}77.2 \\
(11.5)\end{array}$ & 0.430 \\
\hline $\begin{array}{l}\text { Median (IQR) } \\
\text { fasting blood } \\
\text { glucose } \\
(\mathrm{mmol} / \mathrm{l})^{\star \star}\end{array}$ & $\begin{array}{c}4.72(4.34 \\
-5.27)\end{array}$ & $\begin{array}{c}4.99(4.61 \\
-5.44)\end{array}$ & $\begin{array}{c}5.05(4.61 \\
-5.38)\end{array}$ & $\begin{array}{c}5.16(4.77 \\
-5.61)\end{array}$ & $\begin{array}{l}5.27(4.66 \\
-5.51)\end{array}$ & 0 & $\begin{array}{c}4.77(4.44 \\
-5.16)\end{array}$ & $\begin{array}{c}4.88(4.50 \\
-5.27)\end{array}$ & $\begin{array}{c}5.05(4.66 \\
-5.44)\end{array}$ & $\begin{array}{c}5.16(4.77 \\
-5.55)\end{array}$ & $\begin{array}{c}5.22(4.88 \\
-5.55)\end{array}$ & 0 & $\begin{array}{c}1.23(4.60 \\
-5.44)\end{array}$ & $\begin{array}{c}1.27(4.61 \\
-5.38)\end{array}$ & 0.86 \\
\hline Mean (SD) & $167(6)$ & $166(6)$ & $166(6)$ & $166(7)$ & $164(6)$ & 0.003 & $153(7)$ & $153(6)$ & $152(5)$ & $151(5)$ & $150(6)$ & $<0.001$ & $166(6)$ & $152(6)$ & 0.001 \\
\hline
\end{tabular}

$\mathrm{SD}=$ standard deviation; $\mathrm{IQR}=$ =interquartile range; $\mathrm{HDL}=$ high density lipoprotein; $\mathrm{LDL}=$ low density lipoprotein; $\mathrm{BP}=$ blood pressure

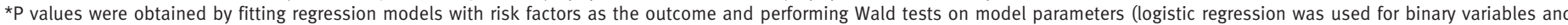
linear regression for continuous variables).

tUsed on a daily basis any time in past 6 months.

$\ddagger$ Consumed on $\geq 10$ days/month any time in past 6 months.

§Physical activity level $\leq 1.69$.

I|k400 g/day.

**Excludes those taking anti-diabetic drugs $(n=54)$

population). P values for trends by socioeconomic position and for differences by sex were obtained by fitting logistic regression models adjusted for age and performing Wald tests on model parameters.

Finally, we projected the burden of these risk factors to the general rural Indian population by estimating the prevalence of each risk factor after direct standardisation by age (grouped in five 10-year bands) and socioeconomic position (low, middle, and high groups, as above) to the population of the national family health survey. ${ }^{35}$ Prevalences in each age and socioeconomic group were calculated and applied to the distributions of age and socioeconomic position in the rural population of the national survey. Standardised prevalences were examined by sex, geographical location (north or south India; south India included the four southern states of Andhra Pradesh, Karnataka Tamil Nadu, and Kerala) and distance to the urban centre (travel time $<4$ hours, $4-12$ hours, $>12$ hours) $\mathrm{P}$ values for differences between standardised prevalences were obtained from $\chi^{2}$ tests. All analyses were conducted with STATA, version 10 (StataCorp, College Station, TX, USA).

\section{Quality assurance}

All instruments and protocols were piloted before the start of the study. Fieldworkers at the four study sites were trained together and standardised at the outset, 
Table 3|Age standardised prevalence of risk factors for non-communicable diseases in rural participants of the Indian Migration Study by socioeconomic position. Values are percentages $(95 \%$ confidence intervals) unless stated otherwise

\begin{tabular}{|c|c|c|c|c|c|c|c|c|c|c|c|}
\hline & \multicolumn{4}{|c|}{ Men's socioeconomic position* } & \multicolumn{4}{|c|}{ Women's socioeconomic position* } & \multicolumn{3}{|c|}{ All socioeconomic position } \\
\hline & $\begin{array}{c}\text { Low } \\
(n=147)\end{array}$ & $\begin{array}{l}\text { Middle } \\
(n=358)\end{array}$ & $\begin{array}{c}\text { High } \\
(n=870)\end{array}$ & P value $†$ & $\begin{array}{c}\text { Low } \\
(n=106)\end{array}$ & $\begin{array}{l}\text { Middle } \\
(n=143)\end{array}$ & $\begin{array}{c}\text { High } \\
(n=359)\end{array}$ & $\mathrm{P}$ value $†$ & $\begin{array}{c}\text { Men } \\
(n=1375)\end{array}$ & $\begin{array}{l}\text { Women } \\
(n=608)\end{array}$ & P value $†$ \\
\hline Smoke tobaccoł & $\begin{array}{c}36.8(29.6 \\
\text { to } 44.1)\end{array}$ & $\begin{array}{c}28.1(23.5 \\
\text { to } 32.7)\end{array}$ & $\begin{array}{c}14.7(12.3 \\
\text { to } 17.1)\end{array}$ & $<0.001$ & $\begin{array}{c}1.2(0.0 \text { to } \\
2.9)\end{array}$ & $\begin{array}{c}1.1 \text { ( } 0.0 \text { to } \\
2.6)\end{array}$ & $\begin{array}{c}0.3(0.0 \text { to } \\
0.8)\end{array}$ & 0.200 & $\begin{array}{l}20.8(18.7 \\
\text { to } 23.0)\end{array}$ & $\begin{array}{c}0.7(0.1 \text { to } \\
1.3)\end{array}$ & $<0.001$ \\
\hline Chew tobacco $\ddagger$ & $\begin{array}{c}23.1(16.2 \\
\text { to } 30.0)\end{array}$ & $\begin{array}{l}25.5(21.0 \\
\text { to } 30.0)\end{array}$ & $\begin{array}{l}23.1(20.3 \\
\text { to } 25.9)\end{array}$ & 0.570 & $\begin{array}{c}7.6(2.8 \text { to } \\
12.5)\end{array}$ & $\begin{array}{c}6.6(3.1 \text { to } \\
10.1)\end{array}$ & $\begin{array}{c}2.0(0.3 \text { to } \\
3.8)\end{array}$ & 0.004 & $\begin{array}{l}23.9(21.7 \\
\text { to } 26.2)\end{array}$ & $\begin{array}{c}3.8(2.4 \text { to } \\
5.2)\end{array}$ & $<0.001$ \\
\hline Alcohol use§ & $\begin{array}{c}33.7(26.2 \\
\text { to } 41.2)\end{array}$ & $\begin{array}{c}26.9(22.3 \\
\text { to } 31.5)\end{array}$ & $\begin{array}{c}20.2(17.5 \\
\text { to } 22.9)\end{array}$ & $<0.001$ & $\begin{array}{c}11.2 \text { (5.6 to } \\
16.8)\end{array}$ & $\begin{array}{c}8.1 \text { (3.4 to } \\
12.9)\end{array}$ & $\begin{array}{c}2.5 \text { (1.0 to } \\
4.1)\end{array}$ & $<0.001$ & $\begin{array}{c}23.5(21.2 \\
\text { to } 25.7)\end{array}$ & $\begin{array}{c}5.4(3.7 \text { to } \\
7.2)\end{array}$ & $<0.001$ \\
\hline $\begin{array}{l}\text { Low physical } \\
\text { activity } \pi\end{array}$ & $\begin{array}{c}65.2(57.5 \\
\text { to } 72.9)\end{array}$ & $\begin{array}{l}72.4(67.8 \\
\text { to } 77.0)\end{array}$ & $\begin{array}{l}72.9(70.0 \\
\text { to } 75.9)\end{array}$ & 0.110 & $\begin{array}{c}66.0(54.5 \\
\text { to } 77.4)\end{array}$ & $\begin{array}{l}73.5(66.0 \\
\text { to } 81.0)\end{array}$ & $\begin{array}{c}76.5(72.0 \\
\text { to } 81.0)\end{array}$ & 0.084 & $\begin{array}{l}72.1(69.8 \\
\text { to } 74.5)\end{array}$ & $\begin{array}{l}74.6(71.0 \\
\text { to } 78.1)\end{array}$ & 0.340 \\
\hline 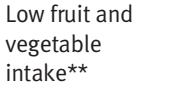 & $\begin{array}{c}81.0(74.5 \\
\text { to } 87.5)\end{array}$ & $\begin{array}{c}75.6(71.2 \\
\text { to } 79.9)\end{array}$ & $\begin{array}{c}63.6(60.3 \\
\text { to } 66.8)\end{array}$ & $<0.001$ & $\begin{array}{c}86.6(77.5 \\
\text { to } 95.7)\end{array}$ & $\begin{array}{l}78.5(71.4 \\
\text { to } 85.5)\end{array}$ & $\begin{array}{c}69.9(65.2 \\
\text { to } 74.7)\end{array}$ & $<0.001$ & $\begin{array}{c}68.6(66.2 \\
\text { to } 71.1)\end{array}$ & $\begin{array}{c}74.8(71.3 \\
\text { to } 78.3)\end{array}$ & 0.005 \\
\hline \multicolumn{12}{|l|}{ Overweight†t: } \\
\hline $\mathrm{BMI} \geq 23$ & $\begin{array}{c}11.4(6.4 \text { to } \\
16.5)\end{array}$ & $\begin{array}{c}20.2(16.0 \\
\text { to } 24.4)\end{array}$ & $\begin{array}{c}43.4(40.2 \\
\text { to } 46.7)\end{array}$ & $<0.001$ & $\begin{array}{c}24.4(16.7 \\
\text { to } 32.1)\end{array}$ & $\begin{array}{c}29.8(22.4 \\
\text { to } 37.3)\end{array}$ & $\begin{array}{c}51.0(46.2 \\
\text { to } 55.9)\end{array}$ & $<0.001$ & $\begin{array}{c}33.7(31.2 \\
\text { to } 36.1)\end{array}$ & $\begin{array}{c}41.9(38.1 \\
\text { to } 45.7)\end{array}$ & $<0.001$ \\
\hline $\mathrm{BMI} \geq 25$ & $\begin{array}{c}5.0(1.5 \text { to } \\
8.5)\end{array}$ & $\begin{array}{c}9.9 \text { (6.8 to } \\
13.1)\end{array}$ & $\begin{array}{l}25.4(22.5 \\
\text { to } 28.2)\end{array}$ & $<0.001$ & $\begin{array}{c}13.3(7.2 \text { to } \\
19.4)\end{array}$ & $\begin{array}{c}19.2(12.8 \\
\text { to } 25.7)\end{array}$ & $\begin{array}{c}35.0(30.3 \\
\text { to } 39.7)\end{array}$ & $<0.001$ & $\begin{array}{l}18.8(16.8 \\
\text { to } 20.9)\end{array}$ & $\begin{array}{l}27.7(24.2 \\
\text { to } 31.2)\end{array}$ & $<0.001$ \\
\hline $\begin{array}{l}\text { Abdominal } \\
\text { obesitył‡ }\end{array}$ & $\begin{array}{c}6.6(2.6 \text { to } \\
10.5)\end{array}$ & $\begin{array}{c}12.1 \text { (8.8 to } \\
15.5)\end{array}$ & $\begin{array}{c}28.2(25.3 \\
\text { to } 31.2)\end{array}$ & $<0.001$ & $\begin{array}{c}9.9(4.6 \text { to } \\
15.2)\end{array}$ & $\begin{array}{c}11.6(6.6 \text { to } \\
16.7)\end{array}$ & $\begin{array}{l}23.8(19.5 \\
\text { to } 28.1)\end{array}$ & $<0.001$ & $\begin{array}{l}21.4(19.3 \\
\text { to } 23.6)\end{array}$ & $\begin{array}{l}18.4(15.4 \\
\text { to } 21.4)\end{array}$ & 0.120 \\
\hline $\begin{array}{l}\text { Total:HDL } \\
\text { cholesterol ratio } \\
\geq 4.5\end{array}$ & $\begin{array}{c}23.6(16.8 \\
\text { to } 30.4)\end{array}$ & $\begin{array}{l}23.4(19.0 \\
\text { to } 27.8)\end{array}$ & $\begin{array}{c}38.5(35.3 \\
\text { to } 41.7)\end{array}$ & $<0.001$ & $\begin{array}{c}32.2(24.0 \\
\text { to } 40.3)\end{array}$ & $\begin{array}{c}32.3(24.7 \\
\text { to } 39.9)\end{array}$ & $\begin{array}{c}35.7(30.7 \\
\text { to } 40.7)\end{array}$ & 0.520 & $\begin{array}{c}33.0(30.5 \\
\text { to } 35.5)\end{array}$ & $\begin{array}{c}34.6(30.8 \\
\text { to } 38.3)\end{array}$ & 0.490 \\
\hline $\begin{array}{l}\text { Triglycerides } \\
\geq 1.69 \mathrm{mmol} / \mathrm{l}\end{array}$ & $\begin{array}{c}20.9(14.6 \\
\text { to } 27.1)\end{array}$ & $\begin{array}{c}22.2(17.9 \\
\text { to } 26.6)\end{array}$ & $\begin{array}{c}29.8(26.7 \\
\text { to } 32.9)\end{array}$ & 0.002 & $\begin{array}{c}25.2(15.0 \\
\text { to } 35.4)\end{array}$ & $\begin{array}{c}19.7(13.2 \\
\text { to } 26.2)\end{array}$ & $\begin{array}{c}31.2(26.3 \\
\text { to } 36.0)\end{array}$ & 0.062 & $\begin{array}{c}26.9(24.6 \\
\text { to } 29.3)\end{array}$ & $\begin{array}{c}27.4(23.8 \\
\text { to } 31.0)\end{array}$ & 0.930 \\
\hline Hypertension§§ & $\begin{array}{c}17.6(12.1 \\
\text { to } 23.2)\end{array}$ & $\begin{array}{c}17.1(13.3 \\
\text { to } 20.9)\end{array}$ & $\begin{array}{c}20.8(18.2 \\
\text { to } 23.5)\end{array}$ & 0.130 & $\begin{array}{c}17.8(11.4 \\
\text { to } 24.2)\end{array}$ & $\begin{array}{c}20.5(14.1 \\
\text { to } 26.9)\end{array}$ & $\begin{array}{c}25.3(21.2 \\
\text { to } 29.3)\end{array}$ & 0.025 & $\begin{array}{l}19.5(17.5 \\
\text { to } 21.5)\end{array}$ & $\begin{array}{c}21.9(18.9 \\
\text { to } 24.9)\end{array}$ & 0.120 \\
\hline DiabetesףT & $\begin{array}{c}1.8 \text { (0.0 to } \\
3.9)\end{array}$ & $\begin{array}{c}3.3(1.4 \text { to } \\
5.2)\end{array}$ & $\begin{array}{c}8.0(6.1 \text { to } \\
9.8)\end{array}$ & $<0.001$ & $\begin{array}{c}3.9(0.8 \text { to } \\
6.9)\end{array}$ & $\begin{array}{c}5.2(2.0 \text { to } \\
8.5)\end{array}$ & $\begin{array}{c}5.1(2.9 \text { to } \\
7.2)\end{array}$ & 0.620 & $\begin{array}{c}6.0(4.7 \text { to } \\
7.3)\end{array}$ & $\begin{array}{c}5.1 \text { (3.5 to } \\
6.8)\end{array}$ & 0.470 \\
\hline Underweight ${ }^{\star \star \star}$ & $\begin{array}{c}35.6(27.8 \\
\text { to } 43.5)\end{array}$ & $\begin{array}{c}29.3(24.6 \\
\text { to } 34.0)\end{array}$ & $\begin{array}{c}15.5(13.1 \\
\text { to } 17.8)\end{array}$ & $<0.001$ & $\begin{array}{c}29.8(19.9 \\
\text { to } 39.6)\end{array}$ & $\begin{array}{c}24.2(16.9 \\
\text { to } 31.6)\end{array}$ & $\begin{array}{c}12.9 \text { (9.5 to } \\
16.3)\end{array}$ & $<0.001$ & $\begin{array}{c}21.2(19.1 \\
\text { to } 23.4)\end{array}$ & $\begin{array}{c}17.9(14.8 \\
\text { to } 21.0)\end{array}$ & 0.057 \\
\hline Short stature††† & $\begin{array}{c}36.0(28.1 \\
\text { to } 43.8)\end{array}$ & $\begin{array}{l}19.2(15.2 \\
\text { to } 23.3)\end{array}$ & $\begin{array}{l}16.2(13.7 \\
\text { to } 18.6)\end{array}$ & $<0.001$ & $\begin{array}{l}33.0(21.7 \\
\text { to } 44.3)\end{array}$ & $\begin{array}{l}29.5(21.9 \\
\text { to } 37.0)\end{array}$ & $\begin{array}{l}20.3(16.1 \\
\text { to } 24.6)\end{array}$ & 0.010 & $\begin{array}{l}18.9(16.8 \\
\text { to } 21.0)\end{array}$ & $\begin{array}{l}24.1(20.7 \\
\text { to } 27.5)\end{array}$ & 0.010 \\
\hline
\end{tabular}

$\mathrm{BMI}=$ body mass index $\left(\mathrm{kg} / \mathrm{m}^{2}\right)$; $\mathrm{HDL}=$ high density lipoprotein

*Based on a subset of questions from the standard of living index ${ }^{24}$ : score 0-7=low, 8-13=middle, 14-36=high.

tP values obtained by fitting logistic regression models adjusted for age, and performing Wald tests on model parameters.

fUsed on a daily basis any time in past 6 months.

$\S$ Consumed on $\geq 10$ days/month any time in past 6 months.

TPhysical activity level $\leq 1.69$

**<400 g/day.

t†Using both the Asian and WHO cut-off values for body mass index.

¥¥Waist circumference $>90 \mathrm{~cm}$ in men, $>85 \mathrm{~cm}$ in women.

$\S \S$ Self reported or blood pressure $\geq 140 / 90 \mathrm{~mm} \mathrm{Hg}$.

१ٓS elf reported or fasting blood glucose concentration $\geq 7 \mathrm{mmol} / \mathrm{l}$.

$\star \star \star$ Body mass index $<18.5$.

††+Height $<25$ th centile of rural population of National Family Health Survey. ${ }^{35}$

and subsequently every six months. The anthropometric equipment was calibrated at the start of every clinic. The Cardiac Biochemistry Laboratory was part of the UK National External Quality Assessment programme for quality assurance of the biochemical assays.

\section{RESULTS}

A total of 13695 factory workers or spouses were interviewed, and 4649 (34\%) had rural relatives. Of these, $4277(92 \%)$ factory workers or spouses agreed to participate in the clinic together with their rural relative. By the end of the study period, 2111 (49\%) rural participants had attended the clinics, with a median time from invitation to clinic participation of 87 days (range 1963). Sixty one were excluded from analyses because they reported their place of residence as urban, and a further 67 were excluded because they fell outside the age range of 20-69 years, leaving 1983 rural participants (1375 men and 608 women) for the present analyses. Data were complete for all the variables except physical activity (16 missing), and biochemical assays (six missing).

Table 1 shows the characteristics of the study population. The participants' median ages were 40 and 42 years for men and women respectively. The 1983 participants came from 18 of India's 28 states (roughly 1600 villages), and 1765 (89\%) came from the four large states in which the factories were located, reflecting the migration patterns of workers and their spouses in these factories. The median travel time to the study centre was 6 hours (range 0-52), with a quarter of the participants travelling for over 12 hours (roughly 600 $\mathrm{km}$ in distance). The median travel time was 8 hours 
Table 4 |Comparison of recent surveys of risk factors for non-communicable diseases in rural India with similar case definitions

\begin{tabular}{|c|c|c|c|c|}
\hline Study & Indian Migration Study & $\begin{array}{l}\text { Andhra Pradesh Rural } \\
\text { Health Initiative15 } 16\end{array}$ & Rajasthan Rural Study14 & Rural Haryana Study13 \\
\hline \multicolumn{5}{|l|}{ Methods } \\
\hline Survey year & $2005-7$ & 2005 & 1995 & $1991-5$ \\
\hline Region & $\begin{array}{l}\text { North and south India } \\
\text { (18 states) }\end{array}$ & $\begin{array}{c}\text { South India } \\
\text { (Andhra Pradesh state) }\end{array}$ & $\begin{array}{c}\text { North India } \\
\text { (Rajasthan state) }\end{array}$ & $\begin{array}{c}\text { North India } \\
\text { (Haryana state) }\end{array}$ \\
\hline Sample frame & Villages from 18 states & 20 villages in one area & 3 villages in one area & Villages in one area \\
\hline Ages (years) & $20-69$ & $\geq 30$ & $\geq 20$ & $35-64$ \\
\hline Sample size & 1983 (1977 blood samples) & 4535 (1085 blood samples) & 3148 (300 blood samples) & 2487 (1190 blood samples) \\
\hline \multicolumn{5}{|c|}{ Results (percentages for men, women) } \\
\hline Current smoker & 42,5 & 45,5 & 51,5 & NA, NA \\
\hline Obesity* & 12,20 & 18,26 & $5,6^{\star}$ & 10,13 \\
\hline Hypertension† & 17,19 & 27,28 & 24,17 & 24,17 \\
\hline Hypercholesterolaemiał & 25,33 & 27,34 & 22,22 & 16,16 \\
\hline Diabetes§ & 4,4 & $14,12^{\star \star}$ & NA & 4,2 \\
\hline
\end{tabular}

*Obesity=body mass index $\geq 25$, except Rajasthan Rural Study with $\mathrm{BMI} \geq 27$.

†Hypertension=self reported or systolic blood pressure $\geq 140 / 90 \mathrm{~mm} \mathrm{Hg}$.

tHypercholesterolaemia=total cholesterol concentration $\geq 5.18 \mathrm{mmol} / \mathrm{l}$.

§Diabetes=self reported or fasting glucose concentration $\geq 7 \mathrm{mmol} / \mathrm{l}$ (in Andhra Pradesh Rural Health Initiative diagnosis based on capillary blood collected by finger prick).

(interquartile range 4-13) for the participants in north India and 5 hours (3-9) for those in south India.

Table 2 shows the distribution of risk factors by age categories. The prevalence of most of the risk factors was higher in older age groups, although for some risk factors it seemed to decline in the oldest age group, possibly due to the small number of participants. Table 3 shows the age standardised prevalence of risk factors by socioeconomic position and sex. Tobacco and alcohol use, low fruit and vegetable intake, and underweight and short stature were more common in the lower socioeconomic groups. Obesity was more prevalent in the higher socioeconomic groups, as were dyslipidaemia and diabetes (in men only) and hypertension (in women only). Tobacco and alcohol use was more common in men, while obesity and low intake of fruit and vegetables was more prevalent in women. The age standardised prevalence of either form of tobacco use (smoked or chewed) was $39.8 \%$ (95\% confidence interval $37.2 \%$ to $42.4 \%$; $\mathrm{n}=543)$ in men and $4.4 \%(2.8 \%$ to $5.9 \% ; \mathrm{n}=27)$ in women $(\mathrm{P}<0.001)$.

The prevalence of self reported medical conditions was low: $1.1 \%(\mathrm{n}=15)$ and $1.8 \%(\mathrm{n}=11)$ of men and women respectively reported heart disease, 5.1\% $(\mathrm{n}=70)$ and $11.5 \%(\mathrm{n}=70)$ reported high blood pressure, $3.4 \%(\mathrm{n}=46)$ and $4.3 \%(\mathrm{n}=26)$ reported diabetes, and $0.4 \%(n=5)$ and $0.8 \%(n=5)$ reported stroke. To investigate whether the low prevalence of risk factors was attributable to social patterning of access to medical care, we examined the prevalence of self reported and newly diagnosed hypertension by the socioeconomic groups. There were clear social trends in the prevalence of self reported hypertension $(3.6 \%(n=9)$ in low socioeconomic group, $5.5 \%(\mathrm{n}=28)$ in medium group, and $8.6 \%(\mathrm{n}=105)$ in high group; $\left.\mathrm{P}_{\text {trend }}=0.001\right)$ but not in the prevalence of newly diagnosed hypertension $(14.3 \%(\mathrm{n}=36), 12.1 \%(\mathrm{n}=60)$, and $14.4 \%(\mathrm{n}=173)$ in low, medium, and high groups; $\left.\mathrm{P}_{\text {trend }}=0.6\right)$. When examined separately by sex, the prevalence of self reported hypertension by socioeconomic group was $5.8 \%(\mathrm{n}=6), 13.6 \%(\mathrm{n}=20)$, and $12.9 \%(\mathrm{n}=46)$ in low, medium, and high groups in women $\left(\mathrm{P}_{\text {trend }}=0.1\right)$; and $2.2 \%(\mathrm{n}=3), 2.3 \%(\mathrm{n}=8)$, and $6.8 \%(\mathrm{n}=59)$ in the three groups in men $\left(\mathrm{P}_{\text {trend }}<0.001\right)$.

The figure shows the prevalence of risk factors standardised to the distribution of age and socioeconomic conditions of the general rural population of India (from the National Family Health Survey ${ }^{35}$ ). The standardisation adjusted the prevalence of risk factors downwards (if the risk factor was more common in upper socioeconomic groups) or upwards (if the risk factor was more prevalent in the lower socioeconomic groups), suggesting that our population was relatively more affluent. The overall patterning of risk factors, however, was largely unchanged: tobacco and alcohol use were more common in men, while obesity was more prevalent in women. Although dyslipidaemia (total:HDL cholesterol ratio $\geq 4.5$ ) was equally prevalent between men and women, women had higher prevalence of both high total cholesterol (levels $\geq 5.18 \mathrm{mmol} / \mathrm{l}$ : $21.1 \%(\mathrm{n}=289)$ men $v 27.8 \% \quad(\mathrm{n}=167)$ women; $\mathrm{P}=0.01)$ and low HDL cholesterol $(31.2 \%$ $(\mathrm{n}=428)$ men with HDL cholesterol $\leq 1.03 \mathrm{mmol} / \mathrm{l} v$ $65.7 \% \quad(\mathrm{n}=398)$ women with HDL cholesterol $\leq 1.28 \mathrm{mmol} / \mathrm{l} ; \mathrm{P}<0.001)$. Apart from underweight, risk factors were generally more prevalent in south Indians compared with north Indians (see web tables 1 and 2 on bmj.com).

\section{DISCUSSION}

The prevalence of risk factors for non-communicable diseases was found to be high among this sample of rural Indians. The prevalence of risk factors generally increased with age. Tobacco and alcohol use and low fruit and vegetable intake were more common in lower 


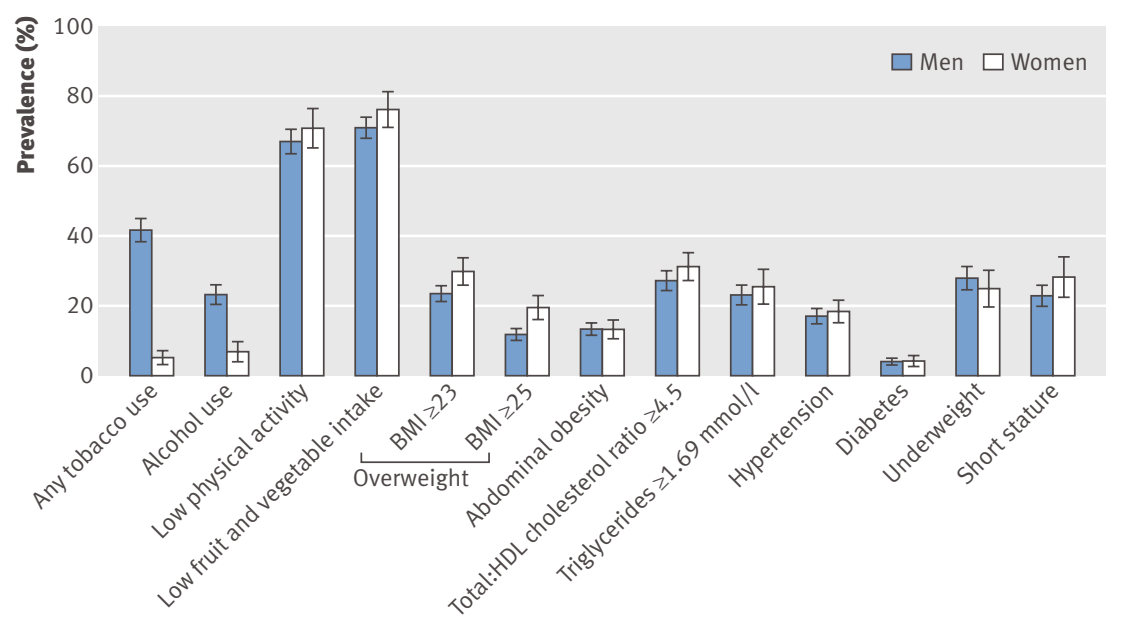

Standardised prevalence of risk factors for non-communicable diseases in rural participants of the Indian Migration Study. (See table 3 for definitions of risk factors)

socioeconomic groups, whereas obesity and some of its related risk factors (dyslipidaemia and diabetes in men and hypertension in women) were more common in higher socioeconomic groups. Tobacco and alcohol use were more common in men, while obesity and low intake of fruits and vegetables were more common in women. Risk factors were more prevalent among south Indians compared with north Indians.

\section{Strengths and limitations of study}

Previous surveys of risk factors for non-communicable diseases among rural Indians have been limited to selected locations (generally within a convenient short distance of an urban centre) or risk factors. ${ }^{513-19}$ This study has examined the distribution of several risk factors among participants from a wide range of rural areas across India using a standardised protocol. Diet and activity data were self reported (in response to questionnaires), which despite our validation studies, have well recognised limitations.

Only half of those eligible participated, raising the possibility of selection bias. It is possible that the rural participants willing to travel to the urban study centre were healthier. Conversely, the chance of a free health check up may have attracted the less healthy. The rural participants were required to have an urban sibling; families generating migrants may be different to those who do not. In addition, having an urban sibling may potentially result in a greater adoption of urban lifestyles, either due to frequent contact with the urban sibling or a higher standard of living from remittances sent home by the migrant. The prevalence of risk factors did not vary much by distance to the study centre. Comparisons of the characteristics of factory participants with and without a participating rural sibling suggested that those who participated were a year younger on average, included a higher proportion of women $(53 \%$ v 60\%; $\mathrm{P}<0.001)$, and reported pre-existing cardiovascular conditions more often $(20 \%$ v $16 \%$; $\mathrm{P}=0.01$ ); however, there was no difference in their smoking prevalence or how far they lived from the study centre (see web table 3 on bmj.com).

We examined the representativeness of the study sample by comparing its sociodemographic characteristics with those of the two main national surveys of India (the 2001 census and the National Family Health Survey NFHS-3 in 2005-6). ${ }^{2335}$ Our study population was more affluent (see web table 4 ). This could be explained by the convenience sampling strategy or differences in age and geographical location of the participants. To account for the relative affluence of our participants, we standardised our data to the age and socioeconomic position of the rural population of the NFHS-3 survey, and these data were also presented. The standardisation altered the estimates somewhat (in the direction expected from the relative affluence of our participants), but did not materially change the patterns. Despite this, the importance of the study limitations (its convenience sampling strategy and low response rate) mean that the findings cannot be regarded as conclusive.

\section{Comparison with previous research}

There are no national studies available for direct comparison. Comparisons with recent surveys from selected locations using similar case definitions suggest broadly similar levels of risk factors, and point towards a temporal rise (table 4). ${ }^{13-16}$ The prevalence of diabetes was lower than that reported in a recent survey from south India ${ }^{16}$ but higher than that reported in the rural component of a national study. ${ }^{19}$ Both these studies used capillary blood samples for diagnosis of diabetes. The prevalence of risk factors in the present study was predictably lower than that reported in recent studies from urban India, although not markedly so; for example, in the urban factory sample to which this study population was related, the prevalence of obesity (body mass index $\geq 25$ ), diabetes, and hypertension in women using similar definitions was found to be $34 \%, 8 \%$, and $25 \%$, compared with $28 \%$, $5 \%$, and $22 \%$ in this study. ${ }^{9-12}$ The higher prevalence of smoking in men and of obesity in women has been noted in other studies. In India obesity and some of its related risk factors tend to be more prevalent in those from higher socioeconomic position (consistent with the early stages of epidemiological transition), ${ }^{51718}$ but recent evidence suggests that this may be changing. ${ }^{3637}$ Tobacco consumption and underweight tend to be commoner in lower socioeconomic positions. ${ }^{38} 39$ Data from this study suggest that the differences in risk factor prevalence may be responsible, at least in part, for the higher prevalence of noncommunicable diseases in south India (although evidence for this widely held belief is limited). ${ }^{2021} \mathrm{How}^{-}$ ever, marked differences in methodologies between studies make comparisons of risk factor data and their interpretation difficult.

\section{Importance of the study findings}

Assessing the patterns of risk factors for non-communicable diseases in rural areas of rapidly growing 


\section{WHAT IS ALREADY KNOWN ON THIS TOPIC}

The burden of risk factors for non-communicable diseases is high in urban India, but recent reports suggest that this trend may be spreading to rural areas

\section{WHAT THIS STUDY ADDS}

Rural participants of the Indian Migration Study showed high levels of risk factors for noncommunicable diseases (particularly tobacco use in men and obesity in women) in the presence of prevalent undernutrition, suggesting that nutrition transition may have spread to parts of rural India

Given the study limitations the results are unlikely to be conclusive, but they strongly warrant careful monitoring and introduction of control measures in rural India

economies such as India is important for several reasons. Firstly, despite rapid urbanisation, most of the population in these countries still live in rural areas. ${ }^{8}$ Contrary to the prevailing belief among policy makers that non-communicable diseases primarily afflict the urban affluent, the burden of non-communicable diseases in rural areas of developing countries is already high and rising. ${ }^{3-5} 15$ Understanding the distributions of the risk factors is vital for planning public health responses. For example, tobacco chewing (a risk factor for oral cancers) is a major problem in north Indian men, but bidis (a form of tobacco smoked predominantly in rural areas) are currently not subject to tax. ${ }^{40}$ Similarly, the coexistence of high levels of underweight and overnutrition require different strategies to address them.

Secondly, unlike the previous epidemic of noncommunicable diseases in developed countries, which was driven mainly by urban migration, the current epidemic in developing countries may also be affected by increasing globalisation-greater interconnectedness of populations ${ }^{67}$ leading to a growing uniformity of lifestyles in both urban and rural areas. ${ }^{4142}$ Unlike in urban areas, the prevalence of risk factors for non-communicable diseases in rural areas is less likely to be confounded by the effects of urban migration and may therefore serve as a crude measure of the effects of globalisation.

Finally, such data may contribute to our understanding of disease aetiology, since juxtaposition of the geographical distributions of prevalence data for risk factors and diseases may allow the relative contributions of genes and environment to be explored, such as the supposed higher prevalence of non-communicable diseases in southern Indians being attributed to their genetic predisposition rather than lifestyle. ${ }^{2021}$ Such analyses would be more informative in rural populations because of an expectation of greater genetic homogeneity than in urban populations, which often include migrants from different places.

\section{Conclusions}

This study suggests that nutrition transition (coexistence of overnutrition and undernutrition) may have progressed to some parts of rural India. Because of the convenience sampling design of the study and its low response rate, the results are unlikely to be conclusive. Nevertheless, these data highlight the need for ongoing monitoring of risk factors for noncommunicable disease in rural India. Introduction of prevention and control measures should be carefully considered.

We thank the local investigators, field workers, and participants of the Indian Migration Study.

Contributors: SK, TL, KSR, AVB, MV, AVK, GDS, YBS, and SE helped design the study; all authors helped conduct the study; LR and RG performed the laboratory analyses; LJB analysed the data; SK wrote the first draft of the manuscript, and all authors contributed to its redrafting and have approved the final version. All authors had full access to all of the data in the study and can take responsibility for the integrity of the data and the accuracy of the data analysis. SK is the guarantor of the study. Funding: This work is funded by the Wellcome Trust (grant No GR070797MF). The funder had no role in study design; data collection, analysis, or interpretation; in writing the report, or in the decision to submit the article for publication. The researchers are all independent from the funding source.

Competing interests: All authors have completed the Unified Competing Interest form at www.icmje.org/coi_disclosure.pdf (available on request from the corresponding author) and declare: no support from any organisation for the submitted work other than the funding grant; no financial relationships with any organisations that might have an interes in the submitted work in the previous 3 years; no other relationships or activities that could appear to have influenced the submitted work. Ethical approval: Ethical approval for the study was obtained from the ethics committee of the All India Institute of Medical Sciences, New Delhi. Written informed consent was obtained from the participants. Data sharing: No additional data available.

1 Reddy KS, Shah B, Varghese C, Ramadoss A. Responding to the threat of chronic diseases in India. Lancet 2005;366:1744-9.

2 Fall $\mathrm{CH}$. Non-industrialised countries and affluence. Br Med Bull 2001;60:33-50.

3 Joshi R, Cardona M, lyengar S, Sukumar A, Raju CR, Raju KR, et al. Chronic diseases now a leading cause of death in rural Indiamortality data from the Andhra Pradesh Rural Health Initiative. Int J Epidemiol 2006;35:1522-9.

4 Gajalakshmi V, Peto R. Verbal autopsy of 80,000 adult deaths in Tamilnadu, South India. BMC Public Health 2004;4:47.

5 Ramachandran A, Snehalatha C, Baskar AD, Mary S, Kumar CK, Selvam S, et al. Temporal changes in prevalence of diabetes and impaired glucose tolerance associated with lifestyle transition occurring in the rural population in India. Diabetologia 2004:47:860-5.

6 Beaglehole R, Yach D. Globalisation and the prevention and control of non-communicable disease: the neglected chronic diseases of adults. Lancet 2003;362:903-8.

7 Reddy KS. Cardiovascular diseases in the developing countries: dimensions, determinants, dynamics and directions for public health action. Public Health Nutr 2002; 5:231-7.

8 Sivaramakrishnan KC, Kundu A, Singh BN. Handbook of urbanisation in India. Oxford University Press, 2007.

9 Gupta R, Joshi P, Mohan V, Reddy KS, Yusuf S. Epidemiology and causation of coronary heart disease and stroke in India. Heart 2008;94:16-26.

10 Gupta R. Coronary heart disease epidemiology: the past, present and future. In: Rao GHR, Kakkar V, eds. Coronary artery disease in South Asians: epidemiology, risk factors and prevention. Jaypee Brothers, 2001:6-28.

11 Gupta R, al-Odat NA, Gupta VP. Hypertension epidemiology in India: meta-analysis of 50 year prevalence rates and blood pressure trends. J Hum Hypertens 1996;10:465-72.

12 Reddy KS, Prabhakaran D, Chaturvedi V, Jeemon P, Thankappan KR, Ramakrishnan L, et al. Methods for establishing a surveillance system for cardiovascular diseases in Indian industrial populations. Bull World Health Organ 2006;84:461-9.

13 Prabhakaran D, Chaturvedi V, Shah P, Manhapra A, Jeemon P, Shah B, et al. Differences in the prevalence of metabolic syndrome in urban and rural India: a problem of urbanization. Chronic Illn 2007;3:8-19.

14 Gupta R, Prakash H, Gupta VP, Gupta KD. Prevalence and determinants of coronary heart disease in a rural population of India. J Clin Epidemiol 1997;50:203-9.

15 Chow CK, Naidu S, Raju K, Raju R, Joshi R, Sullivan D, et al. Significant lipid, adiposity and metabolic abnormalities amongst 4535 Indians from a developing region of rural Andhra Pradesh. Atherosclerosis 2008;196:943-52. 
16 Chow C, Cardona M, Raju PK, Iyengar S, Sukumar A, Raju R, et al. Cardiovascular disease and risk factors among 345 adults in rural India - the Andhra Pradesh Rural Health Initiative. Int J Cardiol 2007;116:180-5.

17 Kutty VR, Balakrishnan KG, Jayasree AK, Thomas J. Prevalence of coronary heart disease in the rural population of Thiruvananthapuram district, Kerala, India. Int J Cardiol 1993;39:59-70.

18 Malhotra P, Kumari S, Kumar R, Jain S, Sharma BK. Prevalence and determinants of hypertension in an un-industrialised rural population of North India. J Hum Hypertens 1999;13:467-72.

19 Sadikot SM, Nigam A, Das S, Bajaj S, Zargar AH, Prasannakumar KM, et al. The burden of diabetes and impaired glucose tolerance in India using the WHO 1999 criteria: prevalence of diabetes in India study (PODIS). Diabetes Res Clin Pract 2004;66:301-7.

20 Ramachandran A, Snehalatha C, Kapur A, Vijay V, Mohan V, Das AK, et al. High prevalence of diabetes and impaired glucose tolerance in India: National Urban Diabetes Survey. Diabetologia 2001;44:1094-101.

21 Gupta R, Gupta VP. Meta-analysis of coronary heart disease prevalence in India. Indian Heart J 1996;48:241-5.

22 Lyngdoh T, Kinra S, Ben-Shlomo Y, Reddy S, Prabhakaran D, Davey Smith G, et al. Sib-recruitment for studying migration and its impact on obesity and diabetes. Emerg Themes Epidemiol 2006;3:2.

23 Registrar General \& Census Commissioner of India. Census of India 2001. Office of the Registrar General of India, 2006.

24 International Institute for Population Sciences (IIPS) and ORC Macro. National family health survey (NFHS-2), 1998-99. IIPS, 2000.

25 Bharathi AV, Kurpad AV, Thomas T, Yusuf S, Saraswathi G, Vaz M. Development of food frequency questionnaires and a nutrient database for the Prospective Urban and Rural Epidemiological (PURE) pilot study in South India: methodological issues. Asia Pac J Clin Nutr 2008;17:178-85.

26 Bharathi AV, Kuriyan R, Kurpad AV, Thomas T, Ebrahim S, Kinra S, et al. Assessment of physical activity using accelerometry, an activity diary, the heart rate method and the Indian migration study questionnaire in south Indian adults. Public Health Nutr 2010;13:47-53.

27 Ainsworth BE, Haskell WL, Whitt MC, Irwin ML, Swartz AM, Strath SJ, et al. Compendium of physical activities: an update of activity codes and MET intensities. Med Sci Sports Exerc 2000;32:498-504.

28 Ross R, Berentzen T, Bradshaw AJ, Janssen I, Kahn HS, Katzmarzyk PT, et al. Does the relationship between waist circumference, morbidity and mortality depend on measurement protocol for waist circumference? Obes Rev 2008;9:312-25.
29 World Health Organization. Diet, nutrition and the prevention of chronic diseases: report of a joint WHO/FAO expert consultation. WHO Technical Report Series 916. WHO, 2003.

30 Appropriate body-mass index for Asian populations and its implications for policy and intervention strategies. Lancet 2004;363:157-63.

31 Ramachandran A, Snehalatha C, Satyavani K, Sivasankari S, Vijay V. Metabolic syndrome in urban Asian Indian adults-a population study using modified ATP III criteria. Diabetes Res Clin Pract 2003;60:199-204

32 Expert Panel on Detection Evaluation and Treatment of High Blood Cholesterol in Adults. Executive summary of the third report of the National Cholesterol Education Program (NCEP) Expert Panel on Detection, Evaluation, and Treatment of High Blood Cholesterol in Adults (Adult Treatment Panel III). JAMA 2001;285:2486-97.

33 Chobanian AV, Bakris GL, Black HR, Cushman WC, Green LA, Izzo JL Jr, et al. The seventh report of the Joint National Committee on Prevention, Detection, Evaluation, and Treatment of High Blood Pressure: the JNC 7 report. JAMA 2003;289:2560-72.

34 Alberti KG, Zimmet PZ. Definition, diagnosis and classification of diabetes mellitus and its complications. Part 1: diagnosis and classification of diabetes mellitus provisional report of a WHO consultation. Diabet Med 1998;15:539-53.

35 International Institute for Population Sciences (IIPS). National family health survey (NFHS-3), 2005-06. IIPS, 2007.

36 Gupta R, Gupta VP, Ahluwalia NS. Educational status, coronary heart disease, and coronary risk factor prevalence in a rural population of India. BMJ 1994;309:1332-6.

37 Reddy KS, Prabhakaran D, Jeemon P, Thankappan KR, Joshi P, Chaturvedi V, et al. Educational status and cardiovascular risk profile in Indians. Proc Natl Acad Sci USA 2007;104:16263-8.

38 Subramanian SV, Nandy S, Kelly M, Gordon D, Davey Smith G. Patterns and distribution of tobacco consumption in India: cross sectional multilevel evidence from the 1998-9 national family health survey. BMJ 2004;328:801-6.

39 Subramanian SV, Davey Smith G. Patterns, distribution, and determinants of under- and overnutrition: a population-based study of women in India. Am J Clin Nutr 2006;84:633-40.

40 World Health Organization. Tobacco or health: a global status report. WHO, 1997.

41 World Health Organization. Globalization, diets and noncommunicable diseases. WHO, 2002.

42 Yach D, Bettcher D. Globalisation of tobacco industry influence and new global responses. Tob Control 2000;9:206-16.

Accepted: 23 July 2010 